\title{
CAPHE: Time-domain and Frequency-domain Modeling of Nonlinear Optical Components
}

\author{
Martin Fiers $^{1,2}$, Thomas Van Vaerenbergh ${ }^{1,2}$, Joni Dambre ${ }^{3}$, Peter Bienstman ${ }^{1,2}$ \\ ${ }^{1}$ Ghent University, Department of Information Technology, Sint-Pietersnieuwstraat 41, 9000 Gent, Belgium \\ ${ }^{3}$ Center for Nano- and Biophotonics (NB-Photonics), Ghent University, Sint-Pietersnieuwstraat 41, 9000 Ghent, Belgium \\ ${ }^{2}$ Ghent University, Department of Electronics and Information Systems, Sint-Pietersnieuwstraat 41, 9000 Gent, Belgium \\ Martin.Fiers@intec.ugent.be
}

\begin{abstract}
We present a tool for the modeling of optical circuits, both in the frequency and in the time domain. The tool is based on the definition of a node, which can have both an instantaneous input-output relation, as well as different state variables (e.g. temperature and carrier density) and differential equations for these states. Furthermore, each node has access to part of its input history, allowing the creation of delay lines or digital filters. Additionally, a node can contain subnodes which can be used to create hierarchical networks. This tool can be used in numerous applications such as frequency-domain analysis of optical ring filters, time-domain analysis of optical amplifiers, microdisks and microcavities. Furthermore, it can be used to study the effect of fabrication errors in nanophotonic integrated circuits. Although we mainly use this tool to model optical circuits, it can also be used to model other classes of dynamical systems, such as neural networks, financial modeling and electronical circuits.

OCIS codes: (130.4310) Nonlinear; (130.6750) Systems; (250.5300) Photonic integrated circuits
\end{abstract}

\section{Introduction}

The typical design cycle for an integrated nanophotonic component consists of first simulating the component, then designing and fabricating a prototype, then measuring the device. The simulation step is particularly important because, when done correctly, it saves a lot of iterations in this design cycle. Depending on the level of detail and how much complexity is required, different simulation techniques are used ranging from Finite Difference Time Domain (FDTD) (e.g. MIT MEEP [1]), Time Domain Traveling Wave (TDTW) [2] (e.g. PicWAVE), Split Step Method (SSM) (e.g. RSoft OptSim) to Coupled Mode Theory and the Transfer Matrix Method (TMM). The first method (FDTD) is the most computationally intense method, and is often used when a single component needs to be studied in-depth. On the other hand, TMM is very fast but less accurate, and is more appropriate for modeling components at the circuit level.

When an optical system contains multiple components, the FDTD method quickly becomes unusable, and highlevel circuit simulation tools are needed. For example, ASPIC [3] is used for calculating the steady-state response of optical circuits, and VPI is mainly used to study the time-domain evolution in optical systems. A different approach is to use the Modified Nodal Analysis (MNA), as used in electronic simulation tools, and extend this to the optical domain by mapping power, wavelength and phase onto voltages and currents, see e.g. [4,5].

Here, we present a different node-based approach. The advantage of our approach is that both the time domain and frequency domain can be modeled in the same framework. Furthermore, all components are represented in a natural way using variables such as the optical field, the temperature and the carrier density, without needing to be mapped on to voltage or current such as in the MNA approach. There are only a few variables per component, which means the simulations are extremely fast compared to FDTD, SSM and TDTW, with the drawback of making approximations. A comparison of the FDTD method and CMT can be found in [6]. Using our approach, components that are approximated as linear and instantaneous (such as splitters, waveguides) can be eliminated from the circuit, effectively reducing the amount of components that need to be simulated in the time-domain simulation. Our tool, called CAPHE, is written in C++ to provide high performance, based on very efficient matrix libraries (eigen3, KLU [7]) and ODE stepping routines (Bulirsch Stoer BS). On top of the C++ core, a Python wrapper is added. Together with scientific libraries for data processing, optimization routines and visualization, this provides a powerful framework to investigate a variety of circuits. Although we mainly use the tool in the optical domain, the node-based approach allows other types of systems to be modeled, such electrical systems and neural networks. 
In section 2 we very briefly sketch the framework. In section 3, we demonstrate how we use CAPHE to optimize an optical filter using an evolutionary algorithm (CMA-ES [8]). Using a variability study, we find that even with state-of-the-art lithography, the fabrication errors are too high for the device to work properly. In the last section, we show how we can eliminate linear, instantaneous nodes to speed up time-domain simulations.

\section{Outline of the framework}

The basic definition of the framework is a Node, as shown in Fig. 1. A detailed overview can be found in [9]. Each Node has $\mathrm{N}$ ports. It has an instantaneous input-output relationship, corresponding to the scatter matrix $\mathbf{S}$ ( $\mathrm{N}$ by $\mathrm{N}$ matrix). This scatter matrix can be wavelength dependent. Furthermore, a Node has a buffer for internal storage of previous time steps, it has states, and Ordinary Differential Equations are defined for these states.

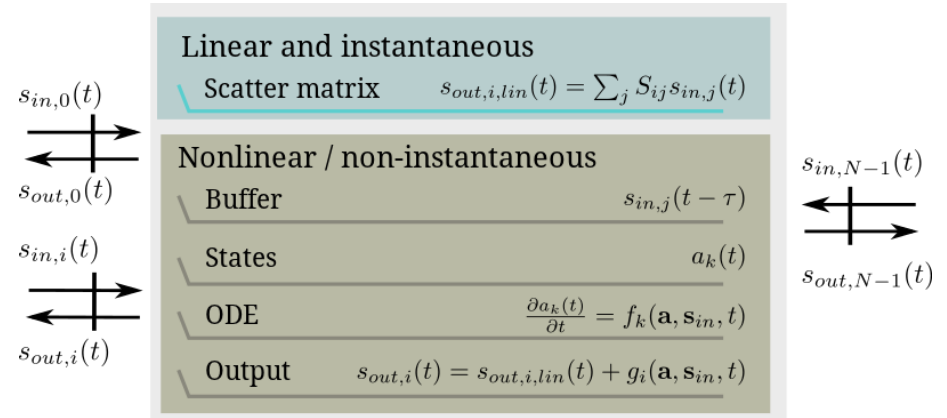

Fig. 1. Basic definition of a Node. It has a linear, instantaneous part (the scatter matrix). A Node can have states, such as the amount of free carriers and temperature, and ODE equations to govern these states. A buffer stores the inputs at previous time steps, these can be used to create delay lines or digital filters.

A circuit is created by linking several nodes together. The total scatter matrix can then be calculated. We use smart matrix solving techniques which allow a scatter matrix of $25000 \times 25000$ to be solved in less than 10 seconds [9], which proves the technique is appropriate for simulating large scale optical systems.

\section{Optimizing a ring resonator filter}

To demonstrate CAPHE in the frequency domain, we optimize a Coupled Resonator Optical Waveguide (CROW):

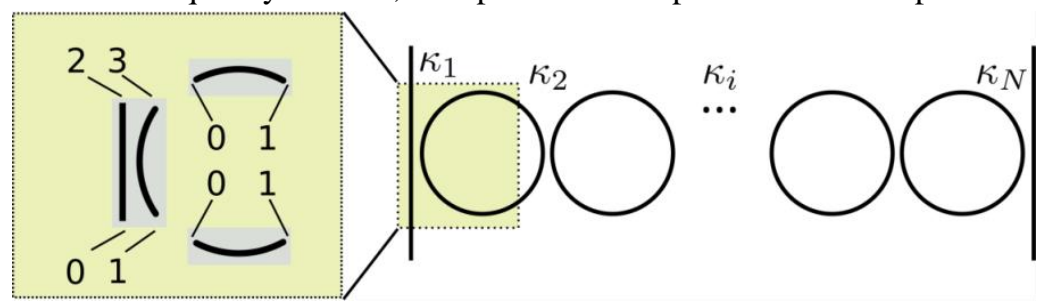

Fig. 2. Coupled Resonator Optical Waveguide. The $\kappa$ are optimized using a genetic algorithm to create a flat filter with a bandwidth of $1 \mathrm{~nm}$. The figure shows how each element contains a directional coupler and two waveguides. The hierarchical model of CAPHE allows to elegantly describe this system.

We use a freely available evolutionary algorithm (CMA-ES [8]) to optimize the $\kappa$ (coupling) between the ring resonators. The target is to design a filter around $\lambda_{\mathrm{r}}=1545 \mathrm{~nm}$ with a pass band of $1 \mathrm{~nm}$, as shown in Fig. 3 .

The ring radius is kept fixed at $5 \mu \mathrm{m}$, and the waveguides loss is $10 \mathrm{~dB} / \mathrm{cm}$. The penalty for the optimization is defined by the root mean square error between the desired and the resulting filter. The result is shown in Fig. 3(left): After 30 iterations the RMSE is 0.012 . Further small improvements will not result in better results due to process variations. To model these process variations we change the radius of the ring. Assume we have a resonance shift due to fabrication errors of about $1 \mathrm{~nm}$ [10]. Using $\Delta \lambda / \lambda=\Delta \mathrm{L} / \mathrm{L}$, this corresponds to a ring radius variation of $20 \mathrm{~nm}$. Fig. 3(right) shows that with these process variations, it is impossible to fabricate a functioning device. Thermal heaters on top of the ring resonators can be used to compensate these variations. Each iteration involved 14 simulations, and the full optimization was done in a few minutes on a personal computer with 2 CPU cores. 

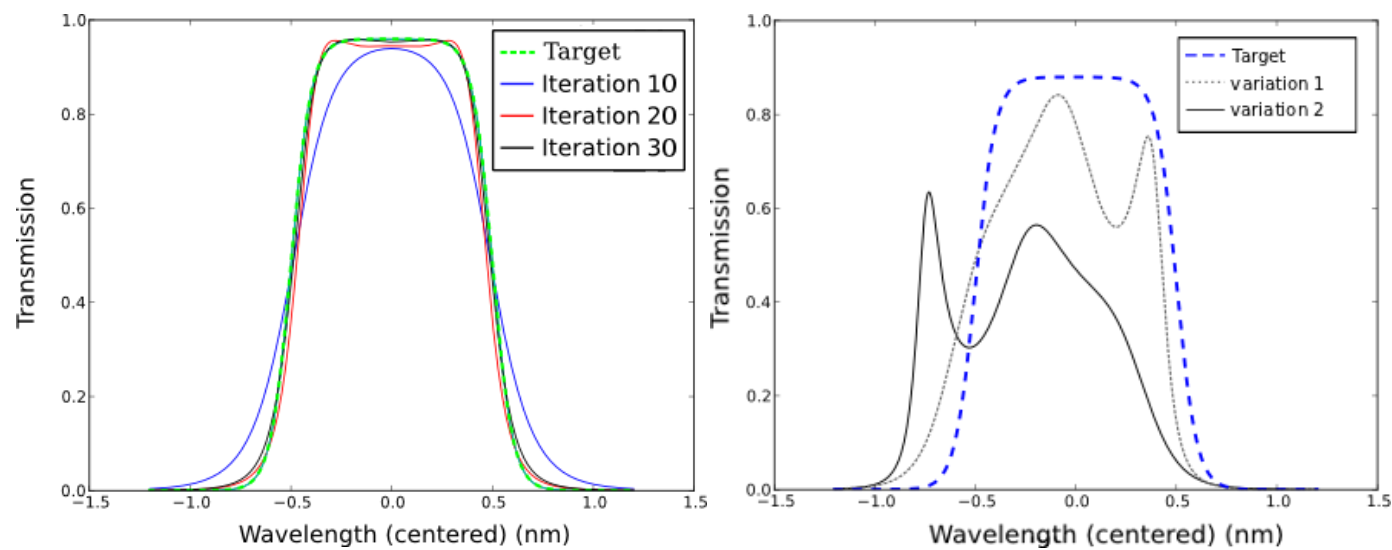

Fig. 3. Left: We optimize the CROW filter (see Fig. 2) for 4 rings, using an evolutionary algorithm. After a few iterations, the target filter is approximated by a RMSE of 0.012 . Right: Simulating the process variations. A ring radius variation of $20 \mathrm{~nm}$ is detrimental for the functionality of the filter.

\section{Speed-up of highly interconnected structures with feedback in time-domain}

In the following example, we simulate a complex network of semiconductor optical amplifiers, linked to each other by passive splitters, combiners and waveguides. By first eliminating these linear nodes, the simulation time and memory requirements for complex circuits are reduced considerably. This is illustrated in Fig. 4.
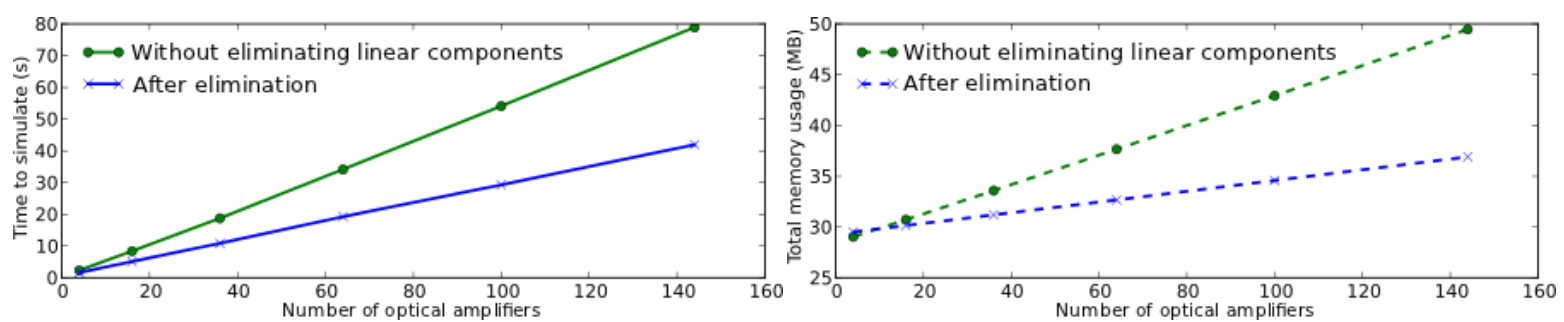

Fig. 4. Simulation of a complex network of optical amplifiers. By eliminating the linear components prior to the time-domain simulation, the simulation time (left) and memory usage (right) are reduced considerably.

\section{References}

[1] A. F. Oskooi, D. Roundy, M. Ibanescu, P. Bermel, J. D. Joannopoulos, and S. G. Johnson, "MEEP: A flexible free-software package for electromagnetic simulations by the FDTD method", Computer Physics Communications 181, 687-702 (2010).

[2] G. Agrawal, Nonlinear Fiber Optics, Third Edition (Optics and Photonics) (2007).

[3] Melloni, A. Canciamilla, A. Ferrari, C. Roncelli, D. Morichetti, F., "A generic design platform for generic photonic foundries", Information Photonics (IP), 2011 ICO International Conference on, Ottawa (2011).

[4] P. Gunupudi, T. Smy, J. Klein, and Z. Jakubczyk, "Self-consistent simulation of opto-electronic circuits using a modified nodal analysis formulation", Advanced Packaging, IEEE Transactions on 33, 979-993 (2010).

[5] T. Smy, P. Gunupudi, S. Mcgarry, and W. N. Ye, "Circuit-level transient simulation of configurable ring resonators using physical models", JOSA B, Vol. 28, pp. 1534-1543 (2011).

[6] B. Maes, M. Fiers and P. Bienstman, "Self-pulsing and chaos in series of coupled nonlinear micro-cavities”,Physical Review B11 7911 (2009).

[7] T. A. Davis and E. Palamadai Natarajan "Algorithm 907: KLU, a direct sparse solver for circuit simulation problems", ACM Transactions on Mathematical Software, vol 37, no. 3 (2010).

[8] N. Hansen, "The CMA evolution strategy: a comparing review," in Towards a new evolutionary computation. Advances on estimation of distribution algorithms, J. Lozano, P. Larranaga, I. Inza, and E. Bengoetxea, Springer, pp. 75-102 (2006).

[9] M. Fiers, T. Van Vaerenbergh, K. Caluwaerts, D. Vande Ginste, B. Schrauwen, J. Dambre, and P. Bienstman, "Time-domain and frequencydomain modeling of nonlinear optical components on circuit-level using a node-based approach", Journal of the Optical Society of America B, accepted for publication (2012).

[10] S. Selvaraja, W. Bogaerts, P. Dumon, D. Van Thourhout, and R. Baets, "Subnanometer linewidth uniformity in silicon nanophotonic waveguide devices using cmos fabrication technology", Selected Topics in Quantum Electronics, IEEE Journal of 16, 316-324 (2010). 